\title{
EPIDEMIOLOGICAL PROFILE OF AGED WOMEN WITH BREAST CANCER IN A PRIVATE CLINIC IN CRICIÚMA/SC
}

\author{
Maristela Harder Peters'1, Giorgia Emilia Klering1, Thamyra Manenti Bonfante1 \\ 'Universidade do Extremo Sul Catarinense Criciúma - Criciúma (SC), Brazil.
}

Objectives: Breast cancer is the most common cancer among women, gradually increasing its incidence in the aged population. This study aims to analyze the epidemiological characteristics of patients over 60 years of age with breast cancer in a private clinic in the city of Criciúma, Santa Catarina. Methods: This is a retrospective observational study, with collection of primary and secondary data and a quantitative approach. The sample was obtained through the analysis of 76 medical records and questionnaires, between 2014 and 2018. Results: The mean age of the patients was 70.39 years. The mean age of menarche and menopause was 13.23 and 49.48 years, respectively. Regarding the body mass index, $38.4 \%$ had some degree of obesity. Most elderly women (81.8\%) were non-smokers. Evenly, 92.3\% were non-alcoholics. Most cancers were detected by mammography (39.3\%). Conservative surgery was predominant (74.2\%). The association between radiotherapy and hormone therapy was the predominant adjuvant therapy (48.7\%). Neoadjuvance was performed in 15 patients, 9 of whom underwent neoadjuvant chemotherapy (60.0\%). The most used hormone therapy was anastrozole (88.9\%). The most common side effects related to hormone therapy were isolated muscle pain (35.7\%) and associated hot flushes (21.4\%). There was a predominance of invasive ductal carcinoma (59.2\%) and Luminal A subtype (43.6\%). Conclusion: Aged patients usually present with smaller and less aggressive tumors, mostly submitted to less aggressive treatments. 